\title{
Global Coral Health Levels Analysis Database with Semantic Computing and 5D World Map
}

\author{
Piyaporn NURARAK ${ }^{\mathrm{a}, 1}$, Yasushi KIYOKI ${ }^{\mathrm{a}}$, \\ Petchporn CHAWAKITCHAREON ${ }^{\mathrm{b}}$ and Yasuhiro HAYASHI ${ }^{\mathrm{c}}$ \\ ${ }^{a}$ Graduate School of Media and Governance, Keio University, Japan \\ ${ }^{b}$ Environmental Engineering Association of Thailand, Thailand \\ ${ }^{c}$ Faculty of Data Science, Musashino University, Japan
}

\begin{abstract}
Global warming and climate change affect not only all living things but also many non-living things. Furthermore, those phenomena caused extreme disasters that become impossible to ignore. Coral bleaching is a phenomenon to show ocean warming due to climate change. This paper presents the analysis and visualization of the coral health levels database by using 5D World Map System. Coral health levels are analyzed using a coral-knowledge image that includes coral with a coral health chart. We use image processing and color semantic distance to interpret coral health levels. We have implemented an actual space integration system to access environmental information resources with coral health levels and image analysis that the results have been shown on the 5D World Map System. As for the experiment study, the study areas of coral health levels analysis are located in the ocean close to Thailand's islands as Ko Ha (Five Island), Ko Bon, Ko Hin Ngam, Ko Tarutao, Ko Thalu, and Ko Samaesarn.
\end{abstract}

Keywords. coral health levels, semantic computing, coral health levels database, coral-knowledge image, image processing, global environmental analysis, 5D World Map.

\section{Introduction}

Global warming and climate change affect not only all living things but also many nonliving things. Furthermore, those phenomena caused extreme disasters such as stronger storms, rising seas, and ocean warming that become impossible to ignore. Global warming is very close to us because everything in this world is dependent on each other. That is why everyone on this planet should start to be aware and understand the impacts of the future. Coral bleaching is a phenomenon to show ocean warming due to climate change. Our motivation is promoting healthy reefs by engaging the global community in monitoring coral health and coral bleaching with 5D World Map System. The 5D World Map System is globally utilized as a Global Environmental Semantic Computing System for disaster, natural phenomena, ocean-water analysis with local and global multimedia data resources.

\footnotetext{
${ }^{1}$ Corresponding Author, Graduate School of Media and Governance, Keio University, Shonan Fujisawa Campus, 5322 Endo, Fujisawa, Kanagawa, 252-0882, Japan; Email: pnurarak@sfc.keio.ac.jp.
} 
This paper presents the analysis and visualization of the coral health levels database by using 5D World Map system. Coral health levels are analyzed using an image that includes coral with a coral health chart. We use image processing and color semantic distance to interpret coral health levels. Our environmental-semantic computing system realizes integration and semantic-search among environmental-semantic spaces with coral health levels and image databases. We have implemented an actual space integration system for accessing environmental information resources with coral health levels and image analysis. We clarify the feasibility and effectiveness of our method and system by showing several experimental results for coral health levels analysis databases.

This paper is organized as follows: Section 2 describes the research related to our study. Section 3 introduces the proposed methods of the system. Section 4 shows results and discussion. Section 5 then presents the conclusion. Finally, Section 6 closes with the acknowledgment.

\section{Related Works}

In this section, we introduce the works that related to our study

\subsection{D World Map System}

The 5D World Map System is globally utilized as a global environmental semantic computing system for disaster, natural phenomena, ocean-water analysis with local and global multimedia data resources. This system, based on the semantic associative computing system (MMM) [1], is a collaborative knowledge sharing, analyzing, searching, integrating, and visualizing data, such as images, videos, audio, etc., onto 5D time-series which temporal (1 Dimension), spatial (2-4 Dimension), and semantic (5 Dimension). In addition, this system integrates and visualizes the analyzed results as multi-dimensional axes dynamic historical atlas. The main feature of this system is to dynamically create various context-dependent environmental patterns according to a user's viewpoints. This system supports the composition of images as the image retriever and enables to realize that users find out the images that show the particular situations of the environment [2-8].

\subsection{Coral-Knowledge Image}

Coral-knowledge image [9] in Figure 1 consists of two essential elements:1) Coral image in the center and 2) Coral health chart [10]. The coral health chart is based on the actual colors of bleached and healthy corals, that each color square corresponds to a concentration of symbionts contained in the coral tissue is directly linked to the health of the coral. This chart represents the most common colors of corals and helps our eyes to make an accurate match. The color is divided into four groups $(\mathrm{B}, \mathrm{C}, \mathrm{D}$, and $\mathrm{E})$ and classified into six levels (1-6) for each side. The highest level (level 6) represents the best healthy coral, and the lowest (level 1) means the worst healthy coral. The health status and mortality percentages from the coral health chart are shown in Table 1 [11]. 


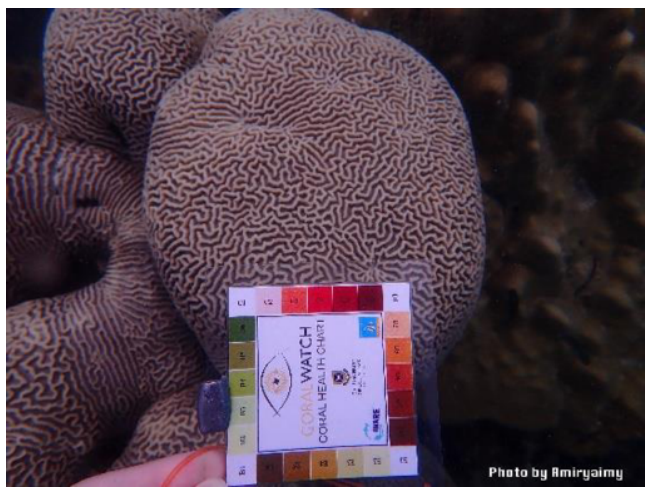

Figure 1. Coral-knowledge image.

Table 1. The health status and mortality percentages from the coral health chart.

\begin{tabular}{cccc}
\hline Level & State of health & Healthy percentage & Mortality percentage \\
\hline 1 & Worst & 16.67 & 83.33 \\
\hline 2 & Poor & 33.33 & 66.67 \\
\hline 3 & Declining & 50.00 & 50.00 \\
\hline 4 & Fair & 66.67 & 33.33 \\
\hline 5 & Good & 83.33 & 16.67 \\
\hline 6 & Best & 100.00 & 0.00 \\
\hline
\end{tabular}

\section{Proposed Methods}

\subsection{Implementation of a prototype system}

This section explains our system's concept of creating an automatic database in semantic space to analyze, interpret, and visualize coral health levels using the 5D World Map system.

The concept of the system is shown in Figure 2:

Step 1: Image data must be coral-knowledge images [8], including coral images and coral health charts.

Step 2: Edge detection is a process to retrieve and detect the coral health chart by using SIFT algorithm (Scale Invariant Feature Transform) to get four corner points and one point at the eye of the coral health chart.[9]

Step 3: Find the chart's color and identify 24 color codes as B1-B6, C1-C6, D1D6, and E1-E6.

Step 4: Getting the color value of coral using k-means clustering to determine the dominant color in coral-knowledge images. The highest score has been chosen to represent the coral color.

Step 5. Interpretation of meaning by color distance computing. We are finding color semantic distance in HSV color space; the result gives the meaning of the coral 
health-level according to the distance ordering of "minimal value" between the color of coral and the closed color code of the chart. Map System.

Step 6. Show the coral health level of the coral-knowledge image in 5D World

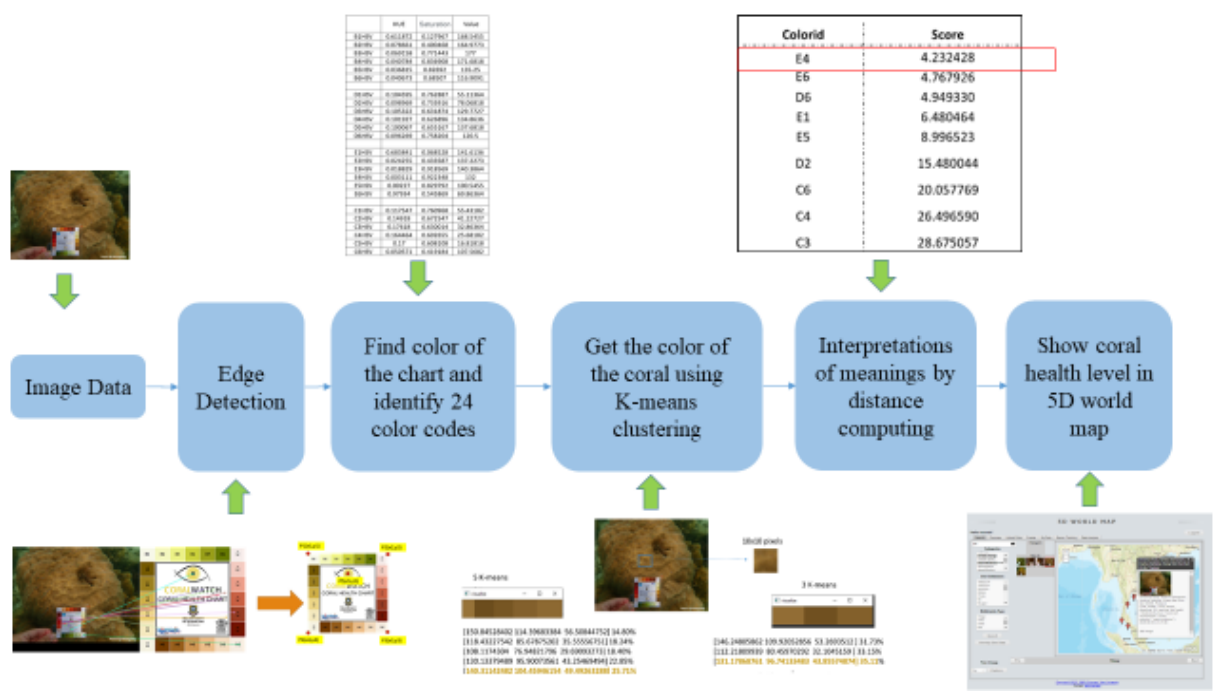

Figure 2. The concept of system.

\subsection{Color semantic distance computing}

We use the color semantic distance calculation to show the color distance between 24 color codes in the coral health chart and coral color value. In HSV color space, we use Eqs. (1) - (4) to find distance between 2 pixels: $\mathrm{P}_{0}\left(\mathrm{~h}_{0}, \mathrm{~s}_{0}, \mathrm{v}_{0}\right)$ and $\mathrm{P}_{1}\left(\mathrm{~h}_{1}, \mathrm{~s}_{1}, \mathrm{v}_{1}\right)$ as follows:

$$
\begin{array}{ll}
\mathrm{dh} & =\min \left(\operatorname{abs}\left(\mathrm{h}_{1}-\mathrm{h}_{0}\right), 360-\mathrm{abs}\left(\mathrm{h}_{1}-\mathrm{h}_{0}\right)\right) / 180.0 \\
\mathrm{ds} & =\mathrm{abs}\left(\mathrm{s}_{1}-\mathrm{s}_{0}\right) \\
\mathrm{dv} & =\mathrm{abs}\left(\mathrm{v}_{1}-\mathrm{v}_{0}\right) / 255.0 \\
\text { distance } & =\sqrt{d h^{2}+d s^{2}+d v^{2}}
\end{array}
$$

When $\mathrm{dh}, \mathrm{ds}$ and $\mathrm{dv}$ are the distance between $\mathrm{P}_{0}$ and $\mathrm{P}_{1}$ in $\mathrm{H}$ (Hue), $\mathrm{S}$ (Saturation), and V (value) color space, respectively. Each of these values will be in the range $[0,1]$.

In the color distance, the smaller result is the higher similarity.

\subsection{K-means Clustering}

Given a set of observations $\left(\mathrm{x}_{1}, \mathrm{x}_{2}, \ldots, \mathrm{x}_{\mathrm{n}}\right)$, where each observation is a d-dimensional real vector, $k$-means clustering aims to partition the $n$ observations into $k(\leq n)$ sets $S=$ $\left\{\mathrm{S}_{1}, \mathrm{~S}_{2}, \ldots, \mathrm{S}_{\mathrm{k}}\right\}$ to minimize the within-cluster sum of squares. 


$$
\underset{s}{\operatorname{argmin}} \sum_{i=1}^{k} \sum_{x \in S_{i}}\left\|x-\mu_{i}\right\|^{2}=\underset{s}{\operatorname{argmin}} \sum_{i=1}^{k}\left|S_{i}\right| \operatorname{Var} S_{i}
$$

Where $\mu_{\mathrm{i}}$ is the mean of points in $\mathrm{S}_{\mathrm{i}}$. This is equivalent to minimizing the pairwise squared deviations of points in the same cluster:

$$
\underset{s}{\operatorname{argmin}} \sum_{i=1}^{k} \frac{1}{2\left|S_{i}\right|} \sum_{x, y \in S_{i}}\|x-y\|^{2}
$$

The equivalence can be deduced from identity

$$
\sum_{x \in S_{i}}\left\|x-\mu_{i}\right\|^{2}=\sum_{x \neq y \in S_{i}}\left(x-\mu_{i}\right)^{T}\left(\mu_{i}-y\right)
$$

In HSV color space, we use k-means clustering to determine the dominant color of the coral in a coral-knowledge image by using 10x10 pixels in the center of the image, as shown in Figure 3.

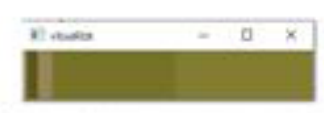

[195.24953789 169.59519409 132.48428835] 1.11\% [91.96934673 90.0532663330 .98190955 ] $4.09 \pi$ [152.06331237 133.84947589 89.95597484] 4.90\% [118.68391109 115.92512415 40.81669158] $42.59 \%$ $[131.73268229 \quad 125.79878472 \quad 50.63237847] 47.32 \%$

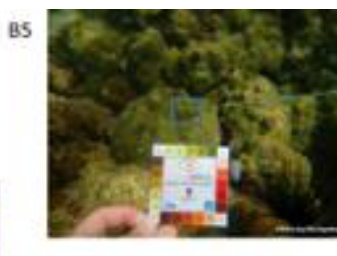

Figure 3. K-means clustering to determine the dominant color.

\subsection{Data Sources}

The data sources that we use in coral health levels analysis are coral-knowledgeimages. The image consists of coral in the center, and the coral health chart can be up, down, left, or right of the coral. All of the coral-knowledge-images have been taken underwater environment. 


\subsection{Description of study areas}

The study areas of coral health levels analysis are located in the ocean close to Thailand's islands as Ko Ha (Five Island), Ko Bon, Ko Hin Ngam, Ko Tarutao, Ko Thalu, and Ko Samaesarn. The descriptions of the coral-knowledge images are shown in Table 2.

Table 2. The description of the coral-knowledge-images.

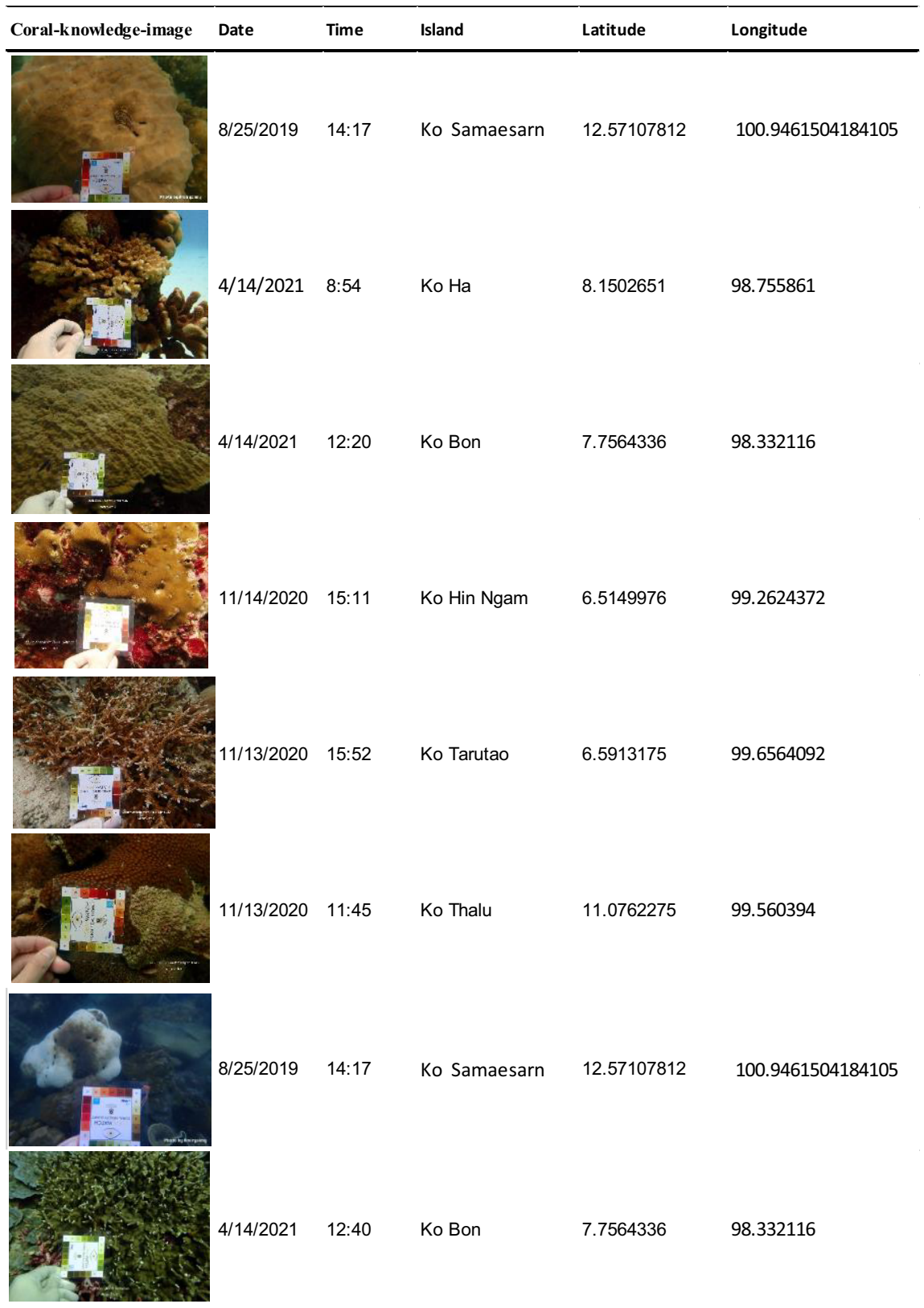




\subsection{Data Structure}

We collected 311 files in CSV form and added semantic and spatiotemporal metadata such as category, location, date, and description for each image data. The data structures are based on 5D World Map System, as shown in Figure 4.

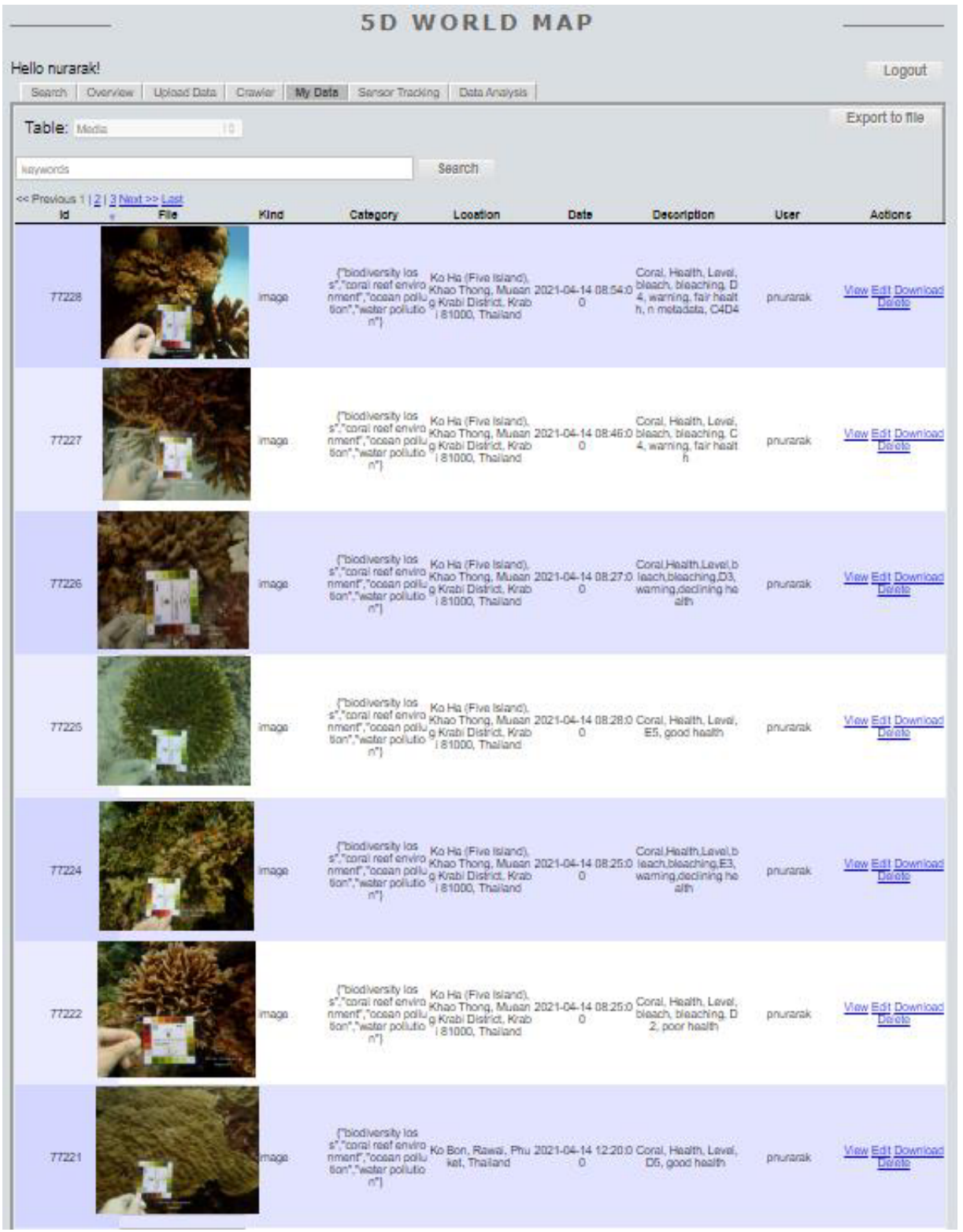

Figure 4. The data structures are based on 5D World Map System. 


\section{Results and Discussion}

To clarify the feasibility and effectiveness of our method and system by (1) showing several experimental results for coral health levels analysis databases and (2) showing several experimental results for coral health levels analysis with a semantic interpretation method and 5D World Map System.

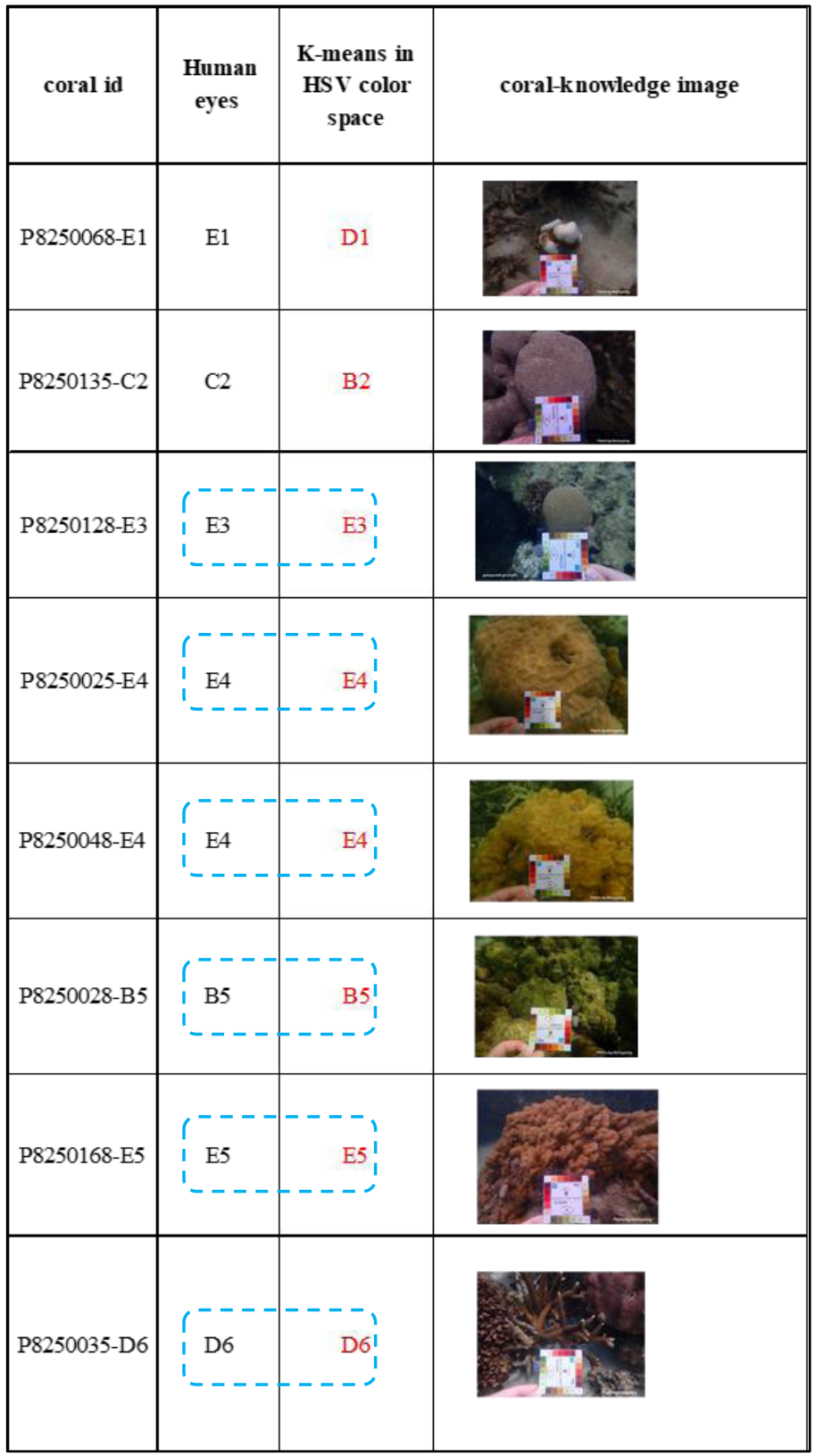

Figure 5. The result of the coral health levels analysis experiments. 


\subsection{Coral Health Levels Analysis Databases}

We have implemented our coral health levels analysis with coral-knowledge image retrieval, using k-means to determine the dominant color in a coral image and color semantic distance computing system for coral-knowledge image datasets. The accuracy of the experiment is $85.0 \%$. Some of the results of the experiment are shown in Figure 5 .

\subsection{Coral Health Levels Analysis with Semantic-Interpretation Method and 5D World Map.}

We realized the mapping and visualization results of coral health levels analysis in 5D World Map System and applied the coral-knowledge image. Our experimental results have shown a semantic associative computing system based on semantic computing in the global environmental analysis shown in Figures 6-9.

(a)

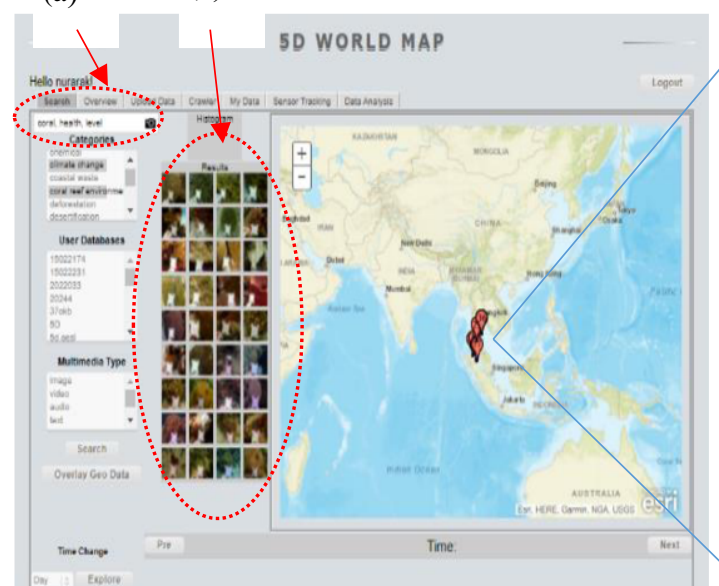

(b)

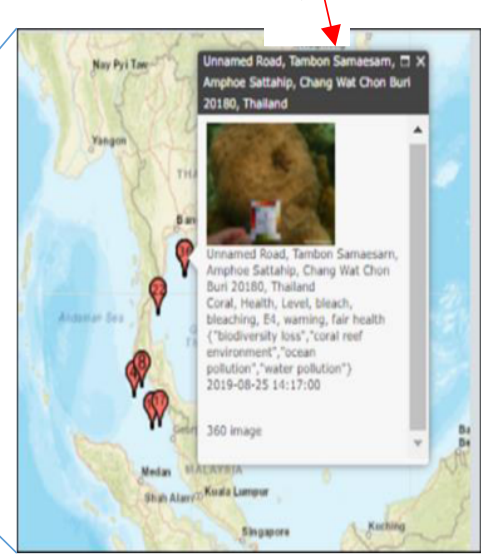

Input keywords $=$ coral health level, Categories $=$ climate change, coral reef environment, ocean pollution

Figure 6. Global coral health levels analysis in 5D world map system:

(a) Keywords search by "coral health levels",

(b) Spatiotemporal analysis (global overview of geographical distribution and the time-series), and (c) Example of coral-knowledge images in climate change, coral reef environment, and ocean pollution categories. 


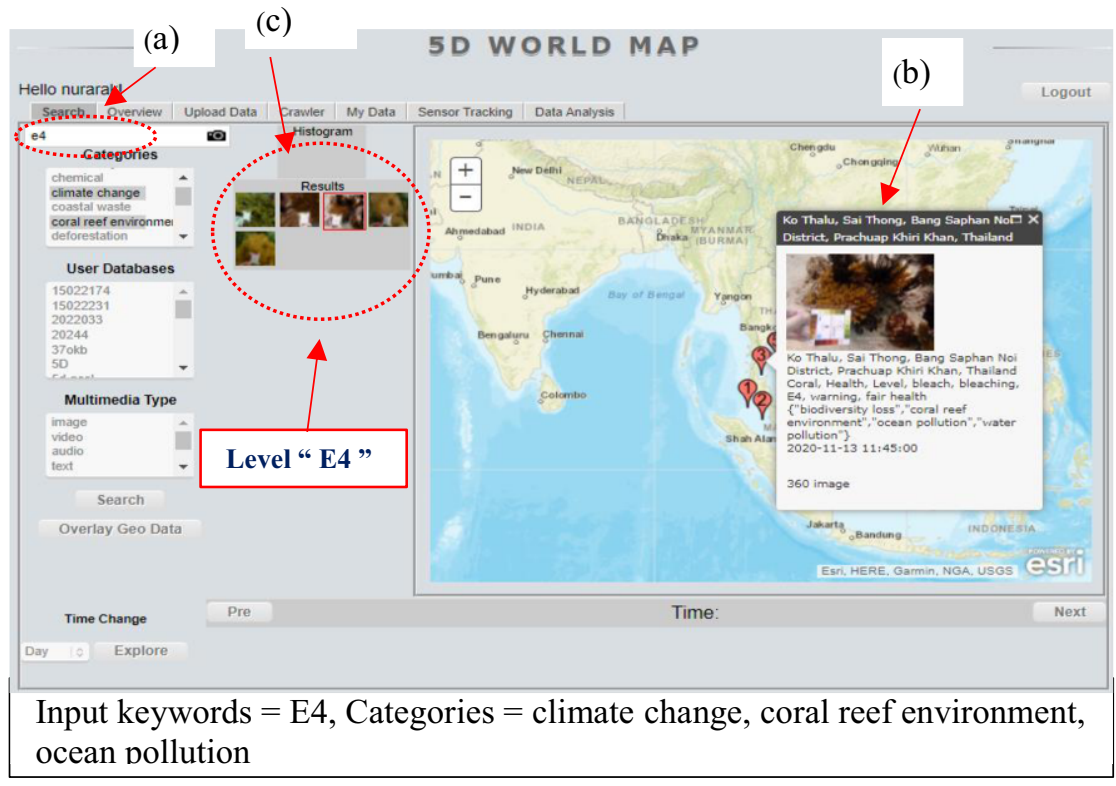

Figure 7. Global coral health levels analysis in 5D World Map System:

(a) Keywords search by coral health levels "E4",

(b) Spatiotemporal mapping in the global overview of geographical distribution. ), and (c) The coral-knowledge images that have health at "E4" ( Fair health) in climate change, coral reef environment, and ocean pollution categories.

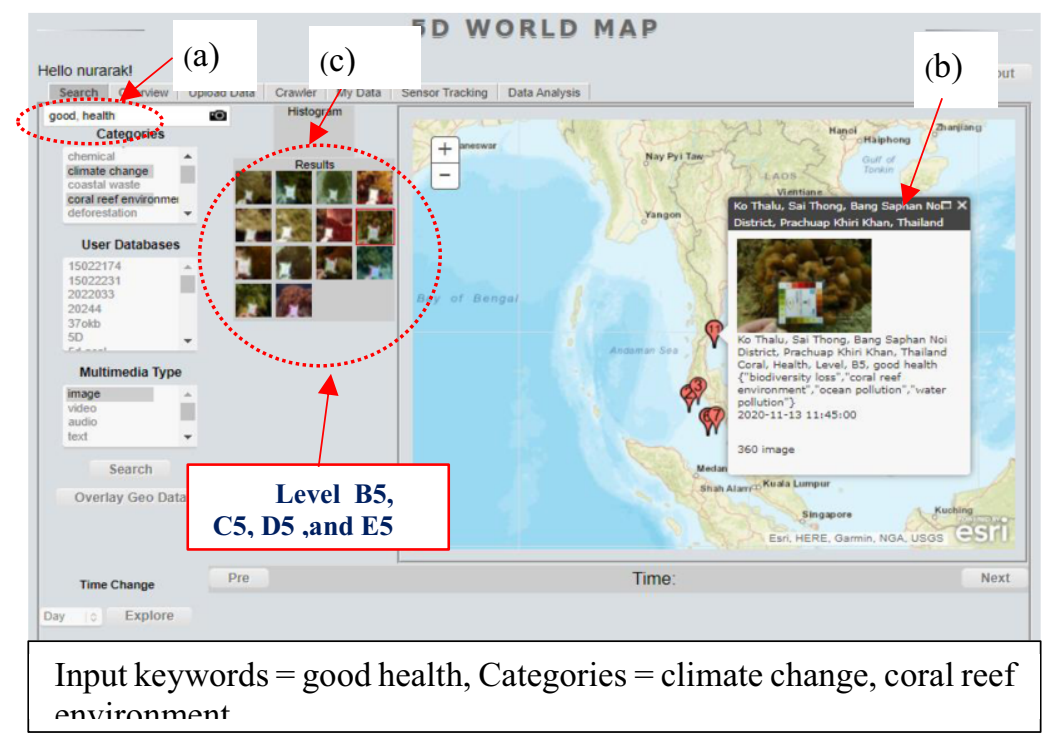

Figure 8. Global coral health levels analysis in 5D world map system:

(a) Keywords search by coral health "Good health",

(b) Spatiotemporal mapping in the global overview of geographical distribution.

(c) The coral-knowledge images that have health at "good health" ( level B5, C5, D5, and E5) in climate change and coral reef environment categories. 


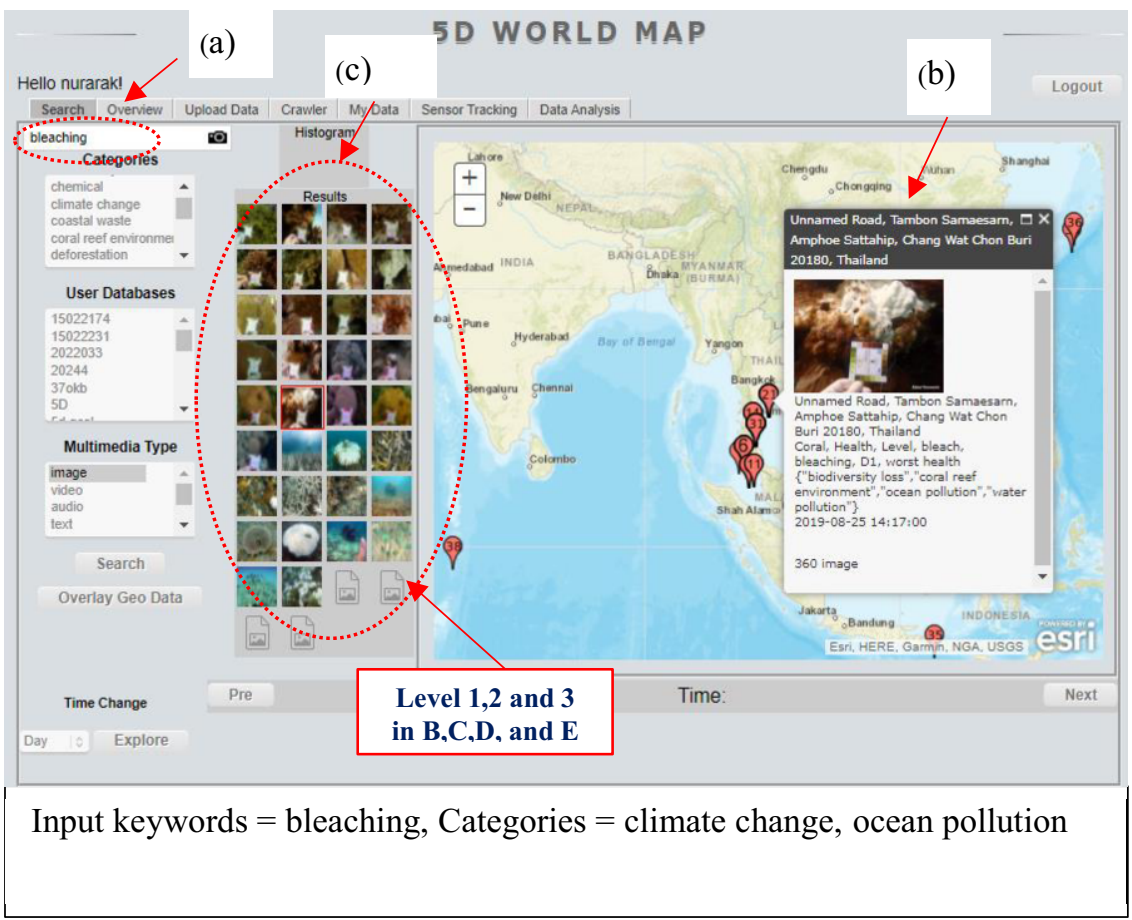

Figure 9. Global coral health levels analysis in 5D world map system:

(a) Keywords search by coral health "bleaching",

(b) Spatiotemporal mapping in the global overview of geographical distribution.

(c) The coral-knowledge images with health levels 1,2 and 3 in B, C, D, and E bleaching in climate change and coral reef environment categories.

\section{Conclusion}

We have presented a new environmental-semantic computing system for coral health levels analysis database and coral-knowledge image spaces with 5D World Map. The main feature of our approach is to realize integration and semantic-search among global environmental-semantic spaces based on the concept of coral health levels analysis space. This system enables creating an automatic coral health level analysis in semantic space for the ocean environment. It makes artificial intelligence vision cognitive functions using image processing with semantic computing instead of the human eye.

As future work, we will extend the coral health levels analysis semantic space realized onto the 5D world map system to global knowledge-sharing on the ocean environment worldwide.

\section{Acknowledgement}

This work is supported by Multimedia Database Laboratory (MDBL), Graduate School of Media and Governance, Keio University. We thank the MDBL members for their 
valuable comments and suggestions. We also appreciate Ms.Veranuch Chawakitchareon for her activities in oceans to take coral photographs.

\section{References}

[1] Kiyoki Y, Kitagawa T, Hayama T. A Metadatabase System for Semantic Image Search by a Mathematical Model of Meaning. SIGMOD Record. $1994 \quad 1$ 12;23(4):34-41. https://doi.org/10.1145/190627.190639

[2] Sasaki S, Kiyoki Y. Analytical Visualization Functions of 5D World Map System for Multi-Dimensional Sensing Data. In Information Modelling and Knowledge Bases XXIX. IOS Press. 2018. p. 71-89. (Frontiers in Artificial Intelligence and Applications). https://doi.org/10.3233/978-1-61499-834-1-71

[3] Kiyoki Y, Chen X, Sasaki S, Koopipat C. Multi-Dimensional Semantic Computing with SpatialTemporal and Semantic Axes for Multi-spectrum Images in Environment Analysis. In Information Modelling and Knowledge Bases XXVII. Vol. 280. IOS Press. 2016. p. 14-30. (Frontiers in Artificial Intelligence and Applications). https://doi.org/10.3233/978-1-61499-611-8-14

[4] Nguyen, D. T. N, Sasaki, S., and Kiyoki, Y. 5D World PicMap: Imagination-based Image Search System with Spatiotemporal Analyzer. Proceeding of The IASTED e-society 2011 Conference, Avila, Spain: 2011. p. 271- 278.

[5] Veesommai C, Kiyoki Y, Sasaki S, Chawakitchareon P. Wide-Area River-Water Quality Analysis and Visualization with 5D World Map System. In Information Modelling and Knowledge Bases XXVII. Vol. 280. IOS Press. 2016. p. 31-41. (Frontiers in Artificial Intelligence and Applications). https://doi.org/10.3233/978-1-61499-611-8-31

[6] Wijitdechakul J, Kiyoki Y, Koopipat C. An environmental-semantic computing system of multispectral imagery for coral health monitoring and analysis. In Information Modelling and Knowledge Bases XXX. IOS Press. 2019. p. 293-311. (Frontiers in Artificial Intelligence and Applications). https://doi.org/10.3233/978-1-61499-933-1-293

[7] Kiyoki Y, Chen X, Veesommai C, Sasaki S, Uraki A, Koopipat C., Chawakitchareon P., Hansuebsai A. An environmental-semantic computing system for coral-analysis in water-quality and multi-spectral image spaces with "Multi-Dimensional World Map". In Information Modelling and Knowledge Bases XXIX. IOS Press. 2018. p. 52-70. (Frontiers in Artificial Intelligence and Applications). https://doi.org/10.3233/978-1-61499-834-1-52

[8] Kiyoki Y, Chawakitchareon P, Rungsupa S, Chen X, Samlansin K. A global \& environmental coral analysis system with spa-based semantic computing for integrating and visualizing ocean-phenomena with "5-dimensional world-map". In Information Modelling and Knowledge Bases XXXII. IOS Press. 2020. p. 76-91. (Frontiers in Artificial Intelligence and Applications). https://doi.org/10.3233/FAIA200821

[9] Nurarak P., Kiyoki Y., Chawakitcharoen P., Hayashi Y. A Coral-Color Analysis System for Observing Environmental Situation and Change with K-means Clustering and Semantic Classification. Thai Environmental Engineering Journal. 2021 Apr 26;35(1):63-73.

[10] Coral Health Chart [Internet]. Australia:CoralWatch; 2021 [cited 2021 Sep 11]; Available from: https://coralwatch.org/index.php/product/coral-health-chart/

[11] Samlansin K., Chawakitchareon P.,Rungsupa S. Effects of Salinity and Nitrate on Coral Health Levels of Acropora sp. Thai Environmental Engineering Journal. 2020 Apr 8;34(1):19-26. 